\title{
COUPLING OF INFRARED RADIATION TO INTERSUBBAND TRANSITIONS IN TUNNELING SUPERLATTICES
}

\author{
C. Nalewajko and M. ZaluŻNy \\ Department of Theoretical Physics, Maria Curie-Skłodowska University \\ Pl. M. Curie-Skłodowskiej 1, 20-031 Lublin, Poland
}

The influence of the interference effects on the intersubband absorption line shape in weakly tunneling superlattices is discussed within the framework of nonlocal effective medium approximation.

PACS numbers: 78.30.Fs, 73.20.Dx

In paper [1] we have shown, neglecting the multiple reflections, that the depolarization effect can very strongly affect the intersubband absorption spectrum of the weakly tunneling superlattices (SL). (We assume that the reader knows Ref. [1].) In this communication we extend the above studies by including interference effects connected with multiple reflections. Our approach is based on the nonlocal effective medium approximation [2,3].

Like in Ref. [1] we consider a system consisting of quantum wells (QWs) with ground states nearly isolated from each other while excited states form a miniband with the width $\Delta$ much smaller than the subband separation. The above condition is fulfilled when we take a set of parabolic QWs where the ground states see a wider barrier than the excited states due to the shape of the potential [4]. The tight-binding approximation gives us the following expression for the direct energy separation between the ground subband and excited miniband

$$
E_{21}\left(k_{z}\right)=E_{21}+(\Delta / 2) \cos \left(k_{z} L_{\mathrm{SL}}\right),
$$

where $E_{21}$ is the subband energy separation of the isolated QW, $L_{\mathrm{SL}}$ is the superlattice period and $k_{z}$ is the $z$ component of the SL wave vector in the first Brillouin zone $\left(-\pi / L_{\mathrm{SL}}<k_{z}<\pi / L_{\mathrm{SL}}\right)$. The numerical value of $\Delta$ depends on the detailed form of the layer potential [4].

Since $L_{\mathrm{SL}}$ is much smaller than the wavelength of the incident radiation, the considered structure can be treated as an effective homogeneous medium (with uniaxial symmetry) described by dielectric tensor $\varepsilon(\omega)[2,3]$

$$
\begin{aligned}
& \varepsilon_{j j}=\varepsilon_{w} \quad j=x, y \\
& \varepsilon_{z z}=\varepsilon_{w}\left[1-\frac{\mathrm{i} 4 \pi \widetilde{\sigma}_{z z}^{(2 \mathrm{D})}(\omega)}{\varepsilon_{w} \omega L_{\mathrm{SL}}}\right]^{-1},
\end{aligned}
$$


where $\varepsilon_{w}$ is the averaged dielectric constant of SL material and $\widetilde{\sigma}_{z z}^{(2 D)}(\omega)$ is the $z z$ component of the modified two-dimensional conductivity tensor describing the current response to the external electric field. It can be calculated using the self-consistent field approach (for details see $[1,5]$ ).

Let us now consider a four layer system corresponding to the typical total internal reflection geometry. The system is formed by semi-infinite medium 0 (GaAs substrate), medium 1 (SL), medium 2 (cap layer) with the thickness $d_{\text {cap }}$ and semi-infinite medium 3 which can be nearly perfect metal or air. In the latter case we assume that the angle of incidence $\varphi$ (in medium 0 ) is larger than the critical angle corresponding to the total internal reflection at 2-3 interface. Therefore, in the absence of the two-dimensional electron gas, all the incident light will be reflected by the structure. Due to the intersubband excitations the energy is removed from the incident beam and the reflection coefficient is reduced to less than unity.

The Fresnel reflection coefficient of the structure $r$ can be calculated using the transfer matrix formalism [6-8]. The absorbance of the structure is connected with $r$ by

$$
A_{\mathrm{SL}}(\omega, \varphi)=1-|r|^{2} .
$$

Figure 1 shows the absorption as a function of photon energy of GaAs QW multilayer system with and without metallic backing. In our numerical calculations we have taken the band parameters of the SL very close to that used in Ref. [4]: electron effective mass $m=0.066 m_{0}, \hbar \tau^{-1}=3 \mathrm{meV}$ ( $\tau$ is the dephasing time

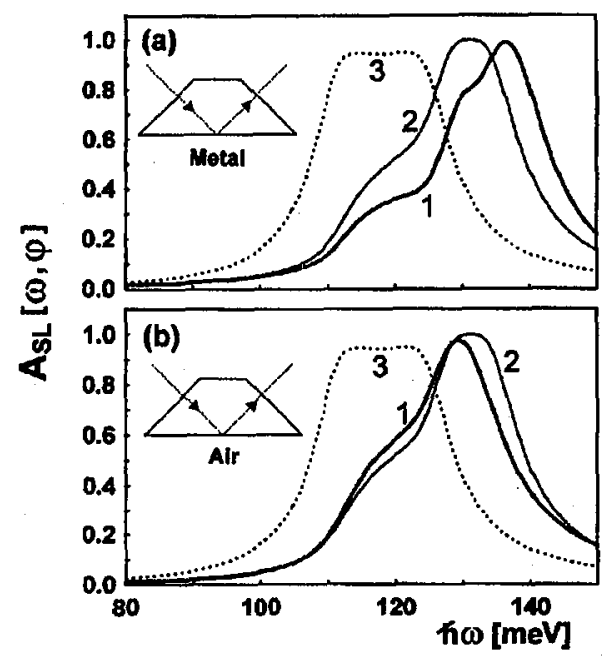

Fig. 1. The spectral characteristics of the structure described in the text: (a) medium 3 is perfect metal; (b) medium 3 is air. Curves labeled by 1 are obtained employing Eq. (4), curves labeled by 2 (3) are obtained employing Eq. (5) and including (neglecting) the depolarization effect. $N=70$ and $d_{\text {cap }}=100 \AA$. 
connected with intersubband transitions), $\varepsilon_{w}=10.9, \Delta=12 \mathrm{meV}, L_{\mathrm{SL}}=132 \AA$, $E_{21}=118 \mathrm{meV}, \varphi=45^{\circ}$ and the electron concentration in a unit area $N_{S}=$ $1.2 \times 10^{12} \mathrm{~cm}^{-2}$. (We assume that only ground subband is occupied by electrons and neglect the difference between the dielectric constants of medium 0,1 , and 2)

For comparison we present also the absorption spectrum obtained neglecting (like in Ref. [1]) the multiple reflections. In this limit (widely used in experimental papers) the absorption is given by

$$
A_{\mathrm{SL}}(\omega, \varphi)=1-\exp [-2 N \alpha(\omega, \varphi)]
$$

where $\alpha(\omega, \varphi)=\left(4 \pi / c \sqrt{\varepsilon_{w}}\right) \operatorname{Re}\left[\widetilde{\sigma}_{z z}^{(2 D)}(\omega)\right] \tan (\varphi) \sin (\varphi)$ and $N$ is the number of QWs in the system.

Our results show that the interference effects connected with the multiple reflections can modify substantially the intersubband absorption line shape. A detailed inspection of Fig. 1 indicates also that this modification (connected mainly with electromagnetic coupling between different QWs) cannot be described (as suggested in Ref. [1]) by a simple renormalization of the Coulomb integral.

\section{References}

[1] M. Załużny, Appl. Phys. Lett. 60, 1486 (1992).

[2] V. Agranovich, V. Kravtsov, Solid State Commun. 55, 85 (1985).

[3] M. Załużny, C. Nalewajko, J. Appl. Phys. 81, 3323 (1997).

[4] R.P.G. Karunasiri, K.L. Wang, Superlattices Microstruct. 4, 661 (1988).

[5] T. Ando, A. Fowler, F. Stern, Rev. Mod. Phys. 54, 437 (1982).

[6] M.A. Azzam, N.M. Bashara, Ellipsometry and Polarized Light; North-Holland, Amsterdam 1987.

[7] M. Załużny, C. Nalewajko, to be published.

[8] C. Nalewajko, Ph.D. Thesis, M. Curie Skłodowska University, Lublin 1998 (in Polish). 